\title{
Misjudgement of gefitinib efficacy in patients with central non-small-cell lung cancer due to obstructive atelectasis caused by stereotactic radiotherapy
}

\author{
XUEQIN YANG, YANLI XIONG, HUAN HUANG, BO PENG, \\ ZEJUN ZHOU, MINGFANG XU, YI YANG and DONG WANG \\ Cancer Center, Daping Hospital, Third Military Medical University, Daping, Yuzhong, Chongqing 400042, P.R. China
}

Received December 10, 2013; Accepted April 8, 2014

DOI: $10.3892 / \mathrm{mco} .2014 .284$

\begin{abstract}
Stereotactic body radiation therapy (SBRT) has been reported to be safe and effective for the treatment of central lung cancer, with mostly tolerable early complications. In this study, we report the development of severe obstructive atelectasis as a late complication in two patients with central lung cancer who received SBRT. This obstructive atelectasis interrupted the evaluation of efficacy of the subsequent gefitinib treatment for non-small-cell lung cancer (NSCLC). The two patients received a total dose of 40 Gy encompassing the planning target volume in 10 fractions (5 fractions/week) at 4 Gy per fraction at the central lesions. Obstructive atelectasis occurred when the patients received subsequent gefitinib treatment. Follow-up reviews or positron emission tomography-computed tomography examination of the two patients confirmed that obstructive atelectasis was actually caused by radiotherapy rather than disease progression. Misjudgement of the cause of ostructive atelectasis in one of the cases resulted in premature termination of gefitinib. Therefore, it is crucial to accurately determine the cause of late complications in NSCLC patients receiving sequential SBRT and gefitinib.
\end{abstract}

\section{Introduction}

Lung cancer is one of the most common and lethal types of cancer, accounting for $17 \%$ of the total new cancer cases and approximately one-fourth of the total cancer-related deaths worldwide (1). Non-small-cell lung cancer (NSCLC) includes adenocarcinoma, squamous cell carcinoma and large-cell carcinoma and is responsible for $\sim 85 \%$ of newly diagnosed lung cancer cases (2). Approximately $80 \%$ of lung cancer cases

Correspondence to: Xueqin Yang, Cancer Center, Daping Hospital, Third Military Medical University, 10 Changjiang Zhi road, Daping, Yuzhong, Chongqing 400042, P.R. China

E-mail: yangxueqin@hotmail.com

Key words: non-small-cell lung cancer, gefitinib, stereotactic radiotherapy have already advanced at the time of diagnosis, thus eliminating surgery as a treatment option. Multidisciplinary treatment is currently the main therapeutic approach for advanced NSCLC. The main method to quickly and effectively control tumor growth is a combination of local treatment and systemic therapy (3). However, the clinical outcomes for radiotherapy combined with chemotherapy, the main therapeutic regimen for advanced NSCLC under the traditional multidisciplinary approach, have been disappointing, with a 5-year survival rate of $<20 \%$ (4-6).

With the increase of the application of targeted therapy for lung cancer, the combination of targeted therapy and radiotherapy has also become a popular therapeutic modality. Theoretically, due to its low toxicity, the combination regimen may be an effective approach, particularly suitable for elderly patients and those with additional underlying diseases $(7,8)$.

However, certain specifications that have been traditionally used for chemotherapy may not be applicable to targeted therapy. Unless disease progresses significantly during the course of targeted therapy, the general recommendation is to continue medication without interruption, as treatment termination is likely to result in rapid disease progression (9). Nishie et al (9) reported a statistically significant difference in the median survival of NSCLC patients who continued epidermal growth factor receptor tyrosine kinase inhibitors (EGFR-TKIs) and those who discontinued such therapy after disease progression, indicating that the management strategy of targeted therapy should be differentiated from that of radiotherapy or chemotherapy.

Stereotactic body radiation therapy (SBRT) delivers high doses of radiation to the involved target field and diminishes the radiation fields by reducing the effects of tumor motion for accuracy and precision, thus contributing to a reduction in the volume of irradiation of normal tissues and an increase in the dose delivered to the target field (10). SBRT was recently used for lung cancer in patients for whom surgery is not a suitable option, with an efficacy comparable to that of surgery for early lung cancer (11). Rowe et al (12) expanded SBRT to central lung cancer and reported that the approach was safe and effective, with mostly tolerable early complications. However, late complications were not addressed in that study. In this study, we report the development of severe obstructive atelectasis as 

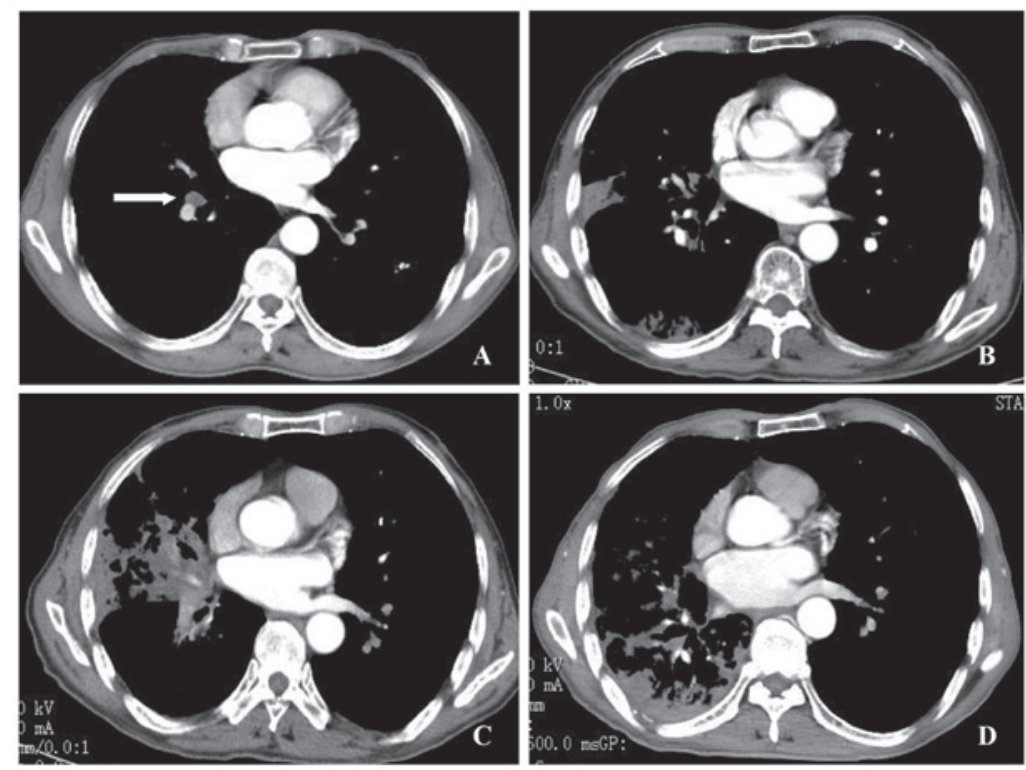

Figure 1. Thoracic computed tomography scan of an 83-year-old man with adenocarcinoma of the right lung. (A) Prior to radiotherapy; (B) 6 months after radiotherapy (mild obstructive pneumonia); (C) 9 months after radiotherapy and 2 weeks after gefitinib initiation (severe obstructive pneumonia in the middle lobe of the right lung); (D) 14 months after radiotherapy (obstructive pneumonia in the middle lobe of the right lung is relieved and obstructive pneumonia in the inferior lobe of the right lung is aggravated). White arrow, location of tumor.
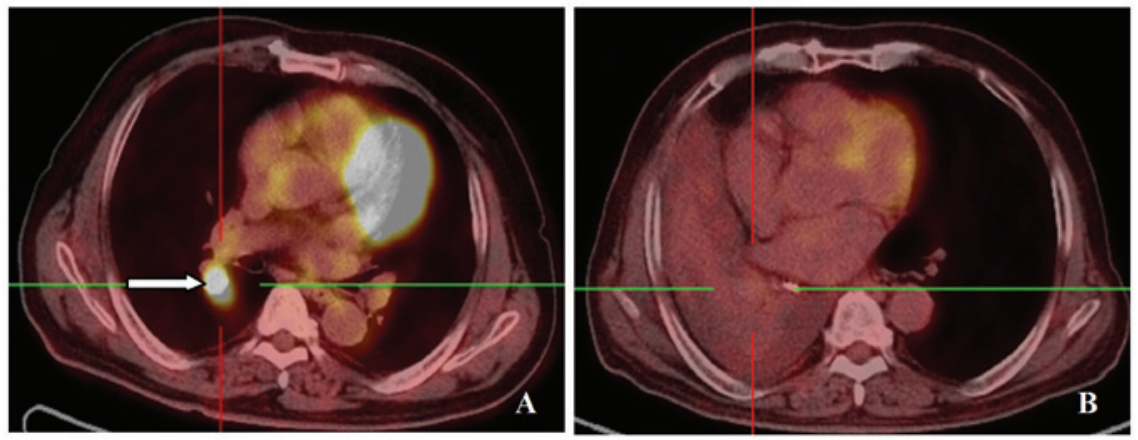

Figure 2. Thoracic PET-CT scan of a 77-year-old man with adenocarcinoma of the right lung. (A) Prior to radiotherapy; (B) 7 months after radiotherapy and 6 months after gefitinib initiation (obstructive atelectasis in the right lobe with no FDG metabolism). White arrow, location of tumor. PET-CT, Positron emission tomography computed tomography; FDG, fluorodeoxyglucose.

a late complication in two patients with central lung cancer who received SBRT. The obstructive atelectasis interrupted the evaluation of the efficacy of the subsequent gefitinib treatment for NSCLC. Informed consent was obtained from the two patients prior to the study. This study was approved by the Ethics Committee of Daping Hospital [(2012)NO.10].

\section{Case reports}

Case 1. An 83-year-old man was diagnosed with stage T2N1M1 adenocarcinoma of the right lung (Fig. 1A). Palliative $\gamma$-ray SBRT was administered to the lesions in the right lower lung, right hilum and left lower lung at doses and fractions as previously described (13). Briefly, computed tomography (CT)-guided simulation was available for delineation of the gross tumor volume (GTV). The planning target volume (PTV) was generated with an additional $0.5 \mathrm{~cm}$ to the GTV in the axial plane and $1.0 \mathrm{~cm}$ in the longitudinal plane. The $\gamma$-SBRT plan included a $50 \%$ isodose line covering $>95 \%$ of the PTV and a 70\% isodose line covering $>90 \%$ of the GTV. A total dose of $40 \mathrm{~Gy}$ was prescribed encompassing the PTV in 10 fractions ( 5 fractions per week) at 4 Gy per fraction and the corresponding dose of $56 \mathrm{~Gy}$ was prescribed encompassing the GTV (5.6 Gy/fraction).

Considering the patient's age, pemetrexed monotherapy was administered 4 times intermittently after radiotherapy. A review conducted at 6 months indicated mild radioactive inflammation of the right lung on CT scan (Fig. 1B). Oral gefitinib was initiated at 9 months post-radiotherapy. Two weeks later, the cough of the patient was exacerbated. A second CT scan revealed obstructive atelectasis in the lateral segment of the right middle lobe (Fig. 1C). The atelectasis was attributed to disease progression and gefitinib was terminated. Given the patient's age, no further treatment options (chemotherapy, radiotherapy, or surgery) were contemplated. A CT scan at 14 months post-radiotherapy revealed that the obstructive atelectasis was mitigated in the lateral segment of the right middle lobe (Fig. 1D). However, the obstructive 
atelectasis in the posterior segment of the right upper lobe, the dorsal segment and the basal segment was aggravated. Metastasis to the contralateral lung was identified 6 months later, indicating disease progression.

Case 2. A 77-year-old man was diagnosed with stage T2N3M0 adenocarcinoma of the right lung. Positron emission tomography (PET)-CT examination revealed nodules in the right lower lobe, enlargement of the lymph nodes at the right hilum and above the left supraclavicular fossa, with increased standardized uptake values (SUV) of fluorodeoxyglucose (Fig. 2A). Palliative $\gamma$-ray SBRT was administered to the right hilum, right lower lobe and lymph nodes on the left supraclavicular fossa with a similar dose schedule to that of Case 1. Following radiotherapy, the patient received bevacizumab, pemetrexed, and nedaplatin. At the 1-month review, the CT scan revealed multiple small nodules in the right middle lobe and disease progression was considered. The patient declined further chemotherapy. Second-line oral gefitinib was initiated, with a decrease in the number of nodules in the right middle lobe 1 month after gefitinib treatment initiation. Partial regression was achieved. Gefitinib was continued for a further 7 months, when the patient experienced worsened post-exertional shortness of breath. A CT scan review revealed obstructive atelectasis in the right lung (Fig. 2B). Progressive disease was contemplated by an outpatient respiratory physician, who recommended terminating gefitinib. However, the PET-CT revealed a mass in the right lower lobe and enlargement of the lymph nodes in the right hilum and left supraclavicular fossa. No increase in SUV was observed in either area, indicating that the activity of the lesions was inhibited. Thus we recommended continuing gefitinib treatment.

\section{Discussion}

As interstitial pneumonia is one of the most severe side effects of EGFR-TKIs and radiotherapy, EGFR-TKIs and concurrent radiotherapy have been used with caution in clinical practice. Wang et al (13) reported only a low incidence rate $(4 \%)$ of third-degree radiation pneumonitis in NSCLC patients receiving EGFR-TKI treatment plus radiotherapy. Okamoto et al (6), however, reported radiation pneumonitis in 2 of 7 stage III unresectable NSCLC patients. Onal et al (14) also reported on a patient who received erlotinib after radiotherapy, which induced radiation pneumonitis. Due to the aforementioned reasons, combination therapy was not prescribed for our two patients. Rather, additional EGFR-TKI treatment was administered sequentially after the completion of radiotherapy.

The main advantage of SBRT is its short radiation time and less damage to the surrounding tissues, resulting in a decreased risk of radiation pneumonitis in lung cancer. Wang et al (15) reported that in 14 patients with advanced NSCLC, SBRT combined with gefitinib resulted in a disease remission rate of up to $57.1 \%$. The median time of disease remission was 8 months, while the 1-year survival rate was $69.6 \%$, suggesting that this regimen may improve local control and disease remission rates with few side effects. Compared to $3 \mathrm{D}-\mathrm{RT}$ or intensity-modulated radiotherapy, SBRT in combination with EGFR-TKIs may be a more suitable treatment option for elderly patients or those with diseases that are a contraindication to radiotherapy, such as chronic obstructive pulmonary disease (16).

However, our two patients with central lung lesions developed severe obstructive atelectasis while on gefitinib after undergoing SBRT, which interrupted our evaluation of gefitinib efficacy. Our analysis indicated that the occurrence of obstructive atelectasis mainly resulted from SBRT for lesions located in the hilum of the lung, which caused the late complication of radioactive bronchial fibrosis, in turn leading to bronchial stenosis. However, such severe obstructive atelectasis cannot exclude the synergistic role of gefitinib. Due to the misjudgement of obstructive atelectasis as a result of disease progression in Case 1, gefitinib was wrongly terminated after 2 weeks, depriving the patient of the opportunity to receive effective treatment. Case 2 was slightly more complicated. The patient did not undergo any gene mutation detection tests. The clinical trial from IPASS (17), suggested that the majority of the patients developed drug resistance within 6-8 months of taking the medication. This patient developed atelectasis exactly 7 months after gefitinib initiation. A PET-CT examination confirmed that there was no progression of the original lesions. Hence, we did not adopt the suggestion by outpatient physicians to discontinue the medication. In fact, Takeda et al (18) reported that, for patients with NSCLC after SBRT, the SUV from PET-CT are of higher diagnostic value regarding local recurrence compared to those from CT.

Our experience with patients who have recently undergone radiotherapy suggests that, if disease progression occurs at the site of radiotherapy during the course of EGFR-TKI treatment, the decision to discontinue the medication should be based on PET-CT rather than CT alone. Furthermore, radioactive atelectasis differs from radiation pneumonitis. If the patient is able to tolerate the condition without severe breathing difficulties, the recommendation is to continue medication. However, our empirical findings must be substantiated and confirmed through further clinical trials.

\section{Acknowledgements}

This study was supported by a grant from the National Natural Science Foundation of China (no. 81272599).

\section{References}

1. Jemal A, Bray F, Center MM, Ferlay J, Ward E and Forman D: Global cancer statistics. CA Cancer J Clin 61: 69-90, 2011.

2. Moran C: Importance of molecular features of non-small cell lung cancer for choice of treatment. Am J Pathol 178: 1940-1948, 2011.

3. Salama JK and Vokes EE: New radiotherapy and chemoradiotherapy approaches for non-small-cell lung cancer. J Clin Oncol 31: 1029-1038, 2013.

4. Pfister DG, Johnson DH, Azzoli CG, et al; American Society of Clinical Oncology: American Society of Clinical Oncology treatment of unresectable non-small-cell lung cancer guideline: update 2003. J Clin Oncol 22: 330-353, 2004

5. Furuse K, Fukuoka M, Kawahara M, et al: Phase III study of concurrent versus sequential thoracic radiotherapy in combination with mitomycin, vindesine, and cisplatin in unresectable stage III non-small-cell lung cancer. J Clin Oncol 17: 2692-2699, 1999.

6. Okamoto I: Overview of chemoradiation clinical trials for locally advanced non-small cell lung cancer in Japan. Int J Clin Oncol 13: 112-116, 2008.

7. Chang CC, Chi KH, Kao SJ, et al: Upfront gefitinib/erlotinib treatment followed by concomitant radiotherapy for advanced lung cancer: a mono-institutional experience. Lung Cancer 73: 189-194, 2011. 
8. Sato Y,Ebara T, Sunaga N, Takahashi T and Nakano T: Interaction of radiation and gefitinib on a human lung cancer cell line with mutant EGFR gene in vitro. Anticancer Res 32: 4877-4881, 2012.

9. Nishie K, Kawaguchi T, Tamiya A, et al: Epidermal growth factor receptor tyrosine kinase inhibitors beyond progressive disease: a retrospective analysis for Japanese patients with activating EGFR mutations. J Thorac Oncol 7: 1722-1727, 2012.

10. Graham JD, Nahum AE and Brada M: A comparison of techniques for stereotactic radiotherapy by linear accelerator based on 3-dimensional dose distributions. Radiother Oncol 22: 29-35, 1991

11. Senan S: Surgery versus stereotactic radiotherapy for patients with early-stage non-small cell lung cancer: More data from observational studies and growing clinical equipoise. Cancer 119: 2668-2270, 2013.

12. Rowe BP, Boffa DJ, Wilson LD, Kim AW, Detterbeck FC and Decker RH: Stereotactic body radiotherapy for central lung tumors. J Thorac Oncol 7: 1394-1399, 2012.

13. Wang J, Xia TY, Wang YJ, et al: Prospective study of epidermal growth factor receptor tyrosine kinase inhibitors concurrent with individualized radiotherapy for patients with locally advanced or metastatic non-small-cell lung cancer. Int J Radiat Oncol Biol Phys 81: e59-e65, 2011.
14. Onal C, Abali H, Koc Z and Kara S: Radiation recall pneumonitis caused by erlotinib after palliative definitive radiotherapy. Onkologie 35: 191-194, 2012.

15. Wang Z, Zhu XX, Wu XH, et al: Gefitinib combined with stereotactic radiosurgery in previously treated patients with advanced non-small cell lung cancer. Am J Clin Oncol: Dec 1, 2012 (Epub ahead of print)

16. Yoshitake T, Nakamura K, Shioyama Y, et al: Stereotactic body radiation therapy for stage I non-small cell lung cancer patients with chronic respiratory insufficiency requiring domiciliary oxygen therapy. Anticancer Res 32: 4041-4044, 2012.

17. Fukuoka M, Wu YL, Thongprasert S, et al: Biomarker analyses and final overall survival results from a phase III, randomized, open-label, first-line study of gefitinib versus carboplatin/paclitaxel in clinically selected patients with advanced non-small-cell lung cancer in Asia (IPASS). J Clin Oncol 29: 2866-2874, 2011.

18. Takeda A, Kunieda E, Fujii H, et al: Evaluation for local failure by 18F-FDG PET/CT in comparison with CT findings after stereotactic body radiotherapy (SBRT) for localized non-small-cell lung cancer. Lung Cancer 79: 248-253, 2013. 